\title{
Metal-Organic Frameworks Help Conducting Polymers Optimize the Efficiency of the Oxygen Reduction Reaction in Neutral Solutions
}

\author{
Matías Rafti, Waldemar A. Marmisollé, and Omar Azzaroni*
}

The oxygen reduction reaction (ORR), where molecular oxygen is electrocatalytically reduced on the electrode surface, plays a decisive role in energy conversion devices..$^{[1,2]}$ Historically, expensive and less earth-abundant noble metals such as platinum have been employed as electrocatalysts for the ORR. ${ }^{[3]}$ Thus, over the last few years considerable research efforts have been focused on the quest for low-cost materials with excellent electrocatalytic activity toward ORR in order to replace expensive metal-based electrocatalysts. ${ }^{[4-6]}$ Within this context, considerable attention was devoted to electrocatalysis at conducting polymers (CPs) electrodes. ${ }^{[7,8]}$ The high conductivity and chemical stability of CPs make them very suitable for electrocatalysis purposes, ${ }^{[9]}$ although they usually exhibit low efficiency compared to other materials. Here, we explore a strategy for improving the electrocatalytic efficiency of a poly(3-aminobenzylamine-co-aniline) (PABA) toward the ORR in neutral solutions by anchoring an electro-inactive metal-organic framework (ZIF-8 MOF) layer on the electroactive film. We demonstrate that the catalytic enhancement arises from the selective uptake and preconcentration of oxygen in the ZIF-8 microporous matrix that ultimately acts as an oxygen reservoir in intimate contact with the electrocatalytic material. Our results show that the rational combination of metal-organic frameworks (MOFs) and electroactive components may be a promising way of preparing low-cost electrocatalytic platforms with optimized functions.

It has been extensively demonstrated that CPs such as polyaniline (PANI) and polypyrrole (PPy) can act as polymeric catalysts capable of promoting the oxygen reduction catalysis on electrode surfaces. As an example, Musiani and co-workers demonstrated the use of PANI as an effective electrocatalytic cathode for oxygen reduction in aqueous sulfuric acid under fuel cell operation conditions displaying good electrochemical reversibility of the redox reaction. ${ }^{[10,11]}$ It is now accepted that among a broad variety of CPs, PANI and PPy have higher electrocatalytic activity in ORR than other polymers. ${ }^{[12]}$ In this

Prof. M. Rafti, Dr. W. A. Marmisollé, Prof. O. Azzaroni Instituto de Investigaciones Fisicoquímicas

Teóricas y Aplicadas (INIFTA)

Departamento de Química

Facultad de Ciencias Exactas

Universidad Nacional de La Plata (UNLP)

CONICET, Boulevard 113 and 64

La Plata 1900, Argentina

E-mail: azzaroni@inifta.unlp.edu.ar

DOI: 10.1002/admi.201600047 respect, the theoretical work of Barzukov and co-workers ${ }^{[7]}$ suggests that the catalysis of the ORR on CPs can be ascribed to the weakening of molecular $\mathrm{O}-\mathrm{O}$ bonds during the chemisorption of oxygen on the polymer surface. For example, in the case of carbon black/PPy composites, the onset potential for ORR $(-0.34 \mathrm{~V})$ is more anodic than that observed in carbon blackcoated electrodes $(-0.60 \mathrm{~V})$. However, the ORR current densities are highly dependent on the loading of the PPy catalyst because of the low oxygen diffusion in the electrode surface. ${ }^{[13]}$ In this regard, one of the major challenges for the optimization of electrochemical energy-conversion devices (e.g., metal-air batteries), is to increase the $\mathrm{O}_{2}$ reduction and evolution efficiencies, thus requiring the development of new effective electrocatalytic interfacial architectures compatible with the operation in aqueous electrolytes using air as the oxygen source.

This point is where MOFs come into the picture as a valuable tool to engineer the oxygen uptake properties of the electrochemical interface in order to increase its concentration on the electrode surface. MOFs represent an emergent and versatile class of microporous materials constituted by an infinite arrangement of metal (or metal cluster) centers coordinated via noncovalent interactions with organic linkers. ${ }^{[14,15]} \mathrm{A}$ great deal of effort has been devoted also to test strategies directed to control MOFs' growth and to synthesize mixed composites and supported films, ${ }^{[16]}$ whose distinctive properties have given rise to an increasing number of interesting applications (e.g., mixed matrix membranes for pervaporation, ${ }^{[17]}$ or thin films for sensor and separation technologies ${ }^{[18]}$ ).

Very recently, Díaz et al. ${ }^{[19]}$ reported a comprehensive work describing the selective gas adsorption of films constituted by ZIF-8 MOF (a member of a subclass of MOFs known as Zeolitic Imidazole Frameworks, constituted of $\mathrm{Co}^{2+}$ or $\mathrm{Zn}^{2+}$ metal ions tetrahedrally coordinated by imidazole-based linkers). It was shown that selectivity of $\mathrm{O}_{2}$ adsorption over $\mathrm{N}_{2}$ is close to 6 , thus confirming that ZIF-8 would undergo preferential uptake of $\mathrm{O}_{2}$ even in the case of atmospheric $\mathrm{O}_{2}: \mathrm{N}_{2}$ composition.

Taking into account these concepts and being aware of the outstanding features of gas storage exhibited by metal-organic frameworks, we took this new paradigm one step further by integrating MOFs onto CPs and using them as oxygen reservoirs sitting atop the electrocatalytic active material. However, it is important to consider that such interfacial architectures require the tailored production and organization of complex matter displaying spatially addressed chemistry using different wet chemical processes. Therefore, to achieve this goal it is important to test strategies for the controlled preparation of multicomponent nanostructures on surfaces. Research efforts 
on this matter are often referred to as "nanoarchitectonics," a term popularized by Ariga and co-workers. ${ }^{[20]}$

The electropolymerization of substituted aniline (ANI) monomers with extra pendant amino group, such as 3-aminobenzylamine (ABA), constitutes a promising strategy toward synthesis of electrode materials which combines the electrical properties of PANI with the chemical richness of the amino moieties. We have recently shown that stable films of copolymers of ANI and ABA (PABA) can be obtained by cyclic voltammetry in acidic solution and then employed in neutral electrolytes. ${ }^{[21]}$ Compared to PANI, the copolymers present increased electroactivity in neutral solution which made them suitable candidates for electrochemical devices operating under biocompatible conditions, such as biofuel cells. ${ }^{[2,23]}$ On the other hand, ZIF-8 constitutes an appealing candidate due to its straightforward synthetic procedure, high surface area, and stability toward hydrolysis in neutral aqueous solutions. In addition, the PABA-modified electrodes offer a great advantage over commonly used carbon substrates, provided that primary amino groups of ABA units can act as efficient nucleation sites for ZIF-8. This ultimately yields an additional control parameter for MOF nucleation and growth. In order to advance in this field, and as a proof of concept, we were interested in demonstrating that the integration of MOFs architectures onto CP films could be a powerful method to enhance the reactant uptake, thus optimizing electrocatalytic functions of the polymer layer. In particular, and as a part of our interest in the use of nanoarchitectonics for advanced applications, we report here the design of a new electrocatalytic interfacial architecture consisting of a CP platform modified with an MOF layer. Our experimental results reveal that the composite material thus obtained exhibits the following attractive functional features: (i) electrocatalytical activity toward ORR in neutral solution, (ii) intrinsic microporosity suitable for selective $\mathrm{O}_{2}$ uptake, and (iii) tunable intergrain mesoporous channels suitable for electrolyte diffusion. We show that the combination of these features into the same interfacial architecture results in an enhancement of the ORR taking place on the polymer layer. This proof of concept can be considered the starting point for further investigations on the rational integration of MOFs and other electrocatalyzers for ORR in neutral solutions.

Details of PABA electrosynthesis on gold electrodes have been presented and discussed in a previous work. ${ }^{[21]}$ Experimental and additional data about electropolymerization, characterization of electroactivity and chemical composition of PABA by X-ray photoelectron spectroscopy (XPS), and scanning electron microscopy (SEM) images of modified substrates are presented in the Supporting Information. Gold-coated quartz crystal microbalance (QCM) sensors were employed as electrodes for electrosynthesis and in situ determinations. After synthesis, the PABA-coated sensors were potentially cycled in pristine $0.5 \mathrm{M} \mathrm{H}_{2} \mathrm{SO}_{4}$ solution. Then, the film was exhaustively washed with $5 \times 10^{-3}$ м 4-(2-hydroxyethyl)-1-piperazineethanesulfonic acid (HEPES) buffer ( $\mathrm{pH} \mathrm{7)} \mathrm{in} 0.1 \mathrm{M} \mathrm{KCl}$ and cyclic voltammetry $(\mathrm{CV})$ was performed in this neutral solution.

The next stage of electrode modification consisted of the assembly of several layers of ZIF-8 MOF from methanolic precursor solutions using the PABA film as the anchoring layer (Supporting Information). The number $(n)$ of deposition cycles
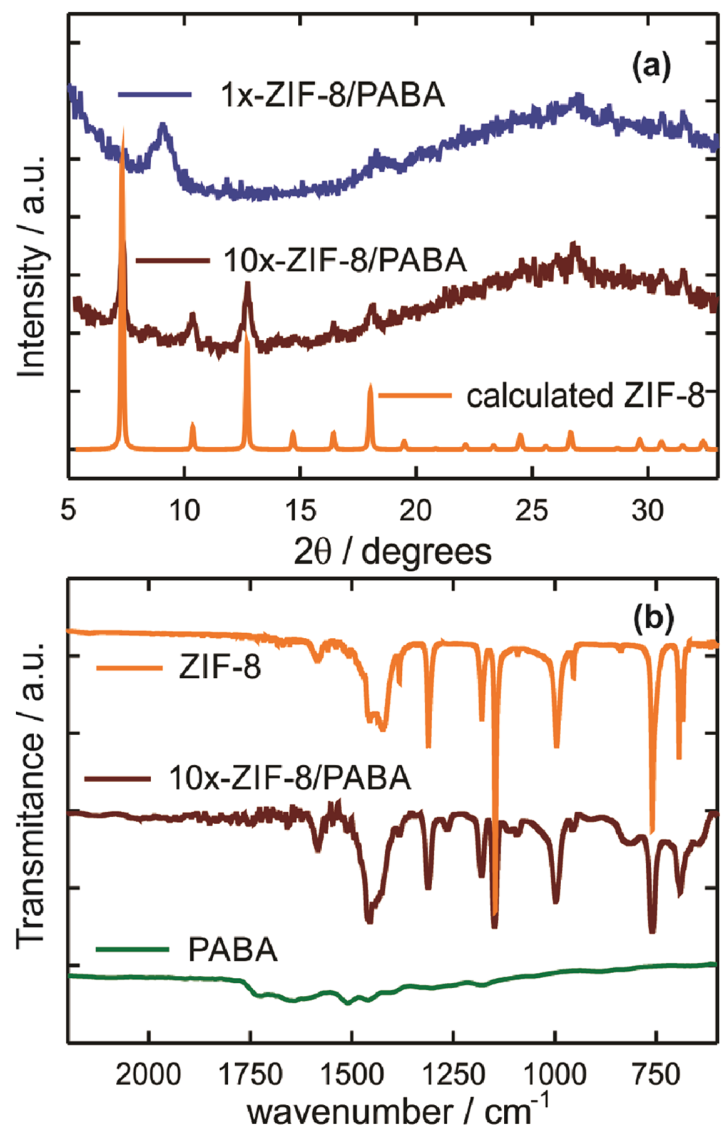

Figure 1. a) Comparison between calculated X-ray diffractograms for ZIF-8, and experimental results for 1x-ZIF-8/PABA and 10x-ZIF-8/PABA. b) Comparison between ATR-FTIR spectra obtained for ZIF-8 powder, 10x-ZIF-8/PABA, and PABA-modified gold substrates.

is indicated here as $n x$-ZIF-8 (see the Supporting Information for detailed information about mass increment versus $n$ ). After washing the electrode with fresh methanol and buffer, CV was again performed in neutral solution. After the electrochemical characterization, the electrodes were washed with water and dried with $\mathrm{N}_{2}$ prior to the structural characterization.

Figure 1a displays a comparison between the calculated ZIF-8 XRD and results obtained for ZIF-8 films growth upon PABA-coated electrodes. The main diffraction peaks corresponding to ZIF-8 crystalline structure are present for the case of 10x-ZIF-8/PABA, thus confirming the nature of the MOF layer obtained. In the case of the 1x-ZIF-8/PABA film, the MOF diffraction peaks are not observed and the diffractogram is dominated by the CP signal. Although PANI is mainly amorphous, broad diffraction peaks at about $9^{\circ}, 15^{\circ}, 22^{\circ}$, and $26^{\circ}$ have been reported, indicating a certain degree of crystalline domains, which could be present also in the copolymer. ${ }^{[24]}$

The identity of the deposited materials was further confirmed by vibrational spectroscopy (Supporting Information). Figure $1 \mathrm{~b}$ shows a comparison of attenuated total reflectance Fourier transform infrared (ATR-FTIR) spectra corresponding to bulk ZIF-8 powder samples, a PABA-coated gold electrode, and a 10x-ZIF-8 film synthesized on PABA-coated Au substrates. PABA spectrum was multiplied by a factor of ten to appreciate 
the bands in the same scale. Owing to the small thickness of the PABA film $(\approx 17 \mathrm{~nm})$, the FTIR bands are weak. They are in the region $1100-1700 \mathrm{~cm}^{-1}$ and are mainly attributed to several aromatic $\mathrm{C}-\mathrm{C}$ and $\mathrm{C}-\mathrm{N}$ stretching modes in benzenic and quinoid units. $^{[25,26]}$ After coating with MOF layers $(\approx 630 \mathrm{~nm})$, PABA features are obscured and the bands appearing on films are in good agreement with bulk ZIF-8 samples. ${ }^{[18,27]}$ Particularly, the band at $1590 \mathrm{~cm}^{-1}$ can be attributed to the $\mathrm{C}=\mathrm{N}$ stretching, while those bands at 1460,1430, and $1390 \mathrm{~cm}^{-1}$ frequencies are related to imidazole ring stretching mode. Bands at 1190, 1150, and $1000 \mathrm{~cm}^{-1}$ can be associated to in-plane ring bending, while bands appearing at 1310 and $950 \mathrm{~cm}^{-1}$ and below $800 \mathrm{~cm}^{-1}$ correspond to out-of-plane ring bending.

The voltammetric response of the PABA-modified Au electrode in air-bubbled buffer is shown in Figure 2a. The voltammetric wave below $-0.2 \mathrm{~V}$ corresponds to the ORR on the CPmodified electrode at a neutral $\mathrm{pH}$.

The reduction of $\mathrm{O}_{2}$ by PANI in acidic media has been shown to occur through the formation of $\mathrm{H}_{2} \mathrm{O}_{2}$ in a two-electron process that oxidizes leucoemeraldine to emeraldine. ${ }^{[10,11]}$ However, the electroreduction of $\mathrm{H}_{2} \mathrm{O}_{2}$ is kinetically inhibited. [10] Additionally, it has been proved theoretically that $\mathrm{O}_{2}$ strongly adsorbs on PANI backbone. ${ }^{[7]}$ However, no catalytic activity of PANI toward oxygen reduction has been observed in neutral solutions, which was mainly attributed to the lower electroactivity of the PANI redox couple in this media. ${ }^{[10]}$ Although in some cases an increase in the peak current was observed, it was

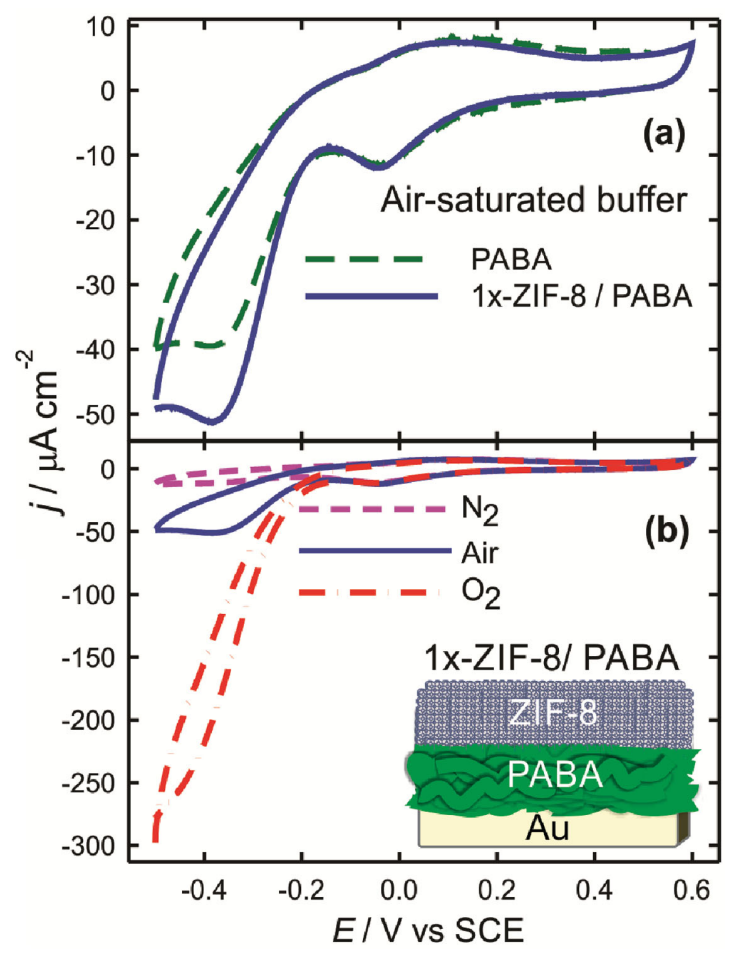

Figure 2. a) Voltammetric response of PABA and 1x-ZIF-8/PABAmodified electrodes in air saturated buffer. b) Comparison of the CV response of a 1x-ZIF-8/PABA-modified electrode at different concentrations of dissolved $\mathrm{O}_{2}$. Bubbled gases are indicted in the plot. Buffer: $0.1 \mathrm{M} \mathrm{KCl} ; 5 \times 10^{-3} \mathrm{~m}$ HEPES; $\mathrm{pH} 7 ; v=0.01 \mathrm{Vs}^{-1}$. attributed to an enhanced $\mathrm{O}_{2}$ adsorption on the polymer layer. ${ }^{[8]}$ In this case, some retardation effects of diffusion through the polymer layer have also been reported. ${ }^{[8]}$ On the other hand, the ORR has also been reported in neutral solution by other PANI derivative, PASA (poly-aminosulfonic acid), which is known to be electrochemically active at $\mathrm{pH} 7 .{ }^{[28]}$ This polymer effectively reduces the overpotential necessary for $\mathrm{O}_{2}$ reduction, which effectively indicates electrocatalysis. There, the observed mechanism was again a two-electron reduction with $\mathrm{H}_{2} \mathrm{O}_{2}$ as product. Based on these previous reports, the ORR on PABA films is likely to occur by this mechanism, promoted by the increased electroactivity of this CP compared with PANI.

As clearly revealed in Figure 2a, after the deposition of an MOF layer on the polymer-coated electrode, the voltammetric wave $\mathrm{O}_{2}$ reduction was notoriously increased. We should note that no enhancement was observed after treatment of the PABA-modified electrode with methanol or methanolic solutions of one of the precursors (see the Supporting Information). This blank experiment confirms that the enhanced activity is not due to chemical effects introduced in the polymer layer as a consequence of any chemical interaction with the MOF precursor solutions. In addition, no shift is observed on the voltammetric peak potential compared with uncovered PABAmodified electrode, which indicates that there is no additional electrocatalytic activity of the composite electrode material. This fact could have been expected as this MOF is not an electroactive material (see the Supporting Information).

Interestingly, instead of blocking the surface, the layer of MOF facilitates the arrival of $\mathrm{O}_{2}$ in the electroactive sites on the CP. This phenomenon in which the reduction current is increased in the presence of the ZIF-8 layer is observed even when thick MOF layers are deposited (see the Supporting Information). Figure $2 \mathrm{~b}$ shows the effect of $\mathrm{O}_{2}$ concentration on the voltammetric response of a 1x-ZIF-8/PABA-modified electrode. Results for 10x-ZIF-8/PABA electrodes are presented in the Supporting Information. The absence of reduction wave in $\mathrm{N}_{2}$ purged solution reaffirms the fact that the current is owing to the ORR whereas the increase of current from air-bubbled to $\mathrm{O}_{2}$-saturated solutions indicates that there is an effective transport of molecular $\mathrm{O}_{2}$ to the active sites on the CP even if a thick layer of MOF is present.

It is important to point out that the MOF layers were stable in the neutral solutions employed as it was verified by the constancy of the initial QCM mass even after potential cycling (see the Supporting Information for further XRD and SEM experiments demonstrating composite stability). It has been reported that ZIF-8 MOF shows high stability toward water steam ${ }^{[29]}$ and it also remains unaltered after soaking in water for 24 h. ${ }^{[30]}$ For practical applications, the hydrothermal stability can be further improved by surface exchange of imidazole ligand by a more hydrophobic derivative. ${ }^{[31]}$

These results suggest that molecular oxygen could be accumulated within the intrinsic hydrophobic microporous network of the ZIF-8 layer, constituting a preconcentration mechanism. In order to evaluate this possibility, the differential uptake of $\mathrm{O}_{2}$ from aqueous solution was measured by QCM. To this end, $\mathrm{N}_{2}$ and $\mathrm{O}_{2}$-saturated buffer solutions were flowed through the QCM cell using dry 1x- and 10x-ZIF-8/PABA modified substrates under atmospheric pressure at room temperature. 
Table 1. Mass increments determined by QCM for different composite electrodes.

\begin{tabular}{lcc}
\hline Composite film & $\begin{array}{c}\Delta m\left[\mu \mathrm{g} \mathrm{cm}^{-2}\right] \text { of MOF } \\
\text { layer deposition }\end{array}$ & $\begin{array}{l}\Delta m\left[\mu \mathrm{g} \mathrm{cm}{ }^{-2}\right] \text { from } \mathrm{N}_{2-} \\
\text { to } \mathrm{O}_{2} \text {-saturated buffer }\end{array}$ \\
\hline 1x-ZIF-8/PABA & 9.8 & 0.36 \\
10x-ZIF-8/PABA & 92.5 & 1.10 \\
\hline
\end{tabular}

The differential uptake of $\mathrm{O}_{2}$ was calculated from the difference between the resonance frequencies in each gas-saturated solution after saturation (flow time $\approx 1 \mathrm{~h}$ ). Table 1 displays uptake values obtained for each substrate. These results support the hypothesis of a selective preconcentration of $\mathrm{O}_{2}$ within the MOF.

It is well known that ZIF-8 yields higher adsorption of $\mathrm{O}_{2}$ than $\mathrm{N}_{2}$ from gas phase, and absolute uptake values are of course highly dependent on temperature and pressure conditions. Moreover, some evidence of a gate-opening transition for the $\mathrm{ZIF}-8 / \mathrm{O}_{2}$ adsorption mechanism was recently reported, ${ }^{[32]}$ thus indicating a considerable interaction between adsorbate molecules and adsorption sites. Room temperature uptakes of $\mathrm{O}_{2}$ and $\mathrm{N}_{2}$ over ZIF-8 nanocrystals grown over electrospun polyurethane have been reported to be 0.29 and $0.19 \mathrm{~cm}^{3} \mathrm{~g}^{-1}$ (STP); ${ }^{[33]}$ whereas Gadipelli et al. have reported somehow higher values for not-supported calcined ZIF-8 for the same conditions $\left(1.56 \mathrm{~cm}^{3} \mathrm{~g}^{-1} \mathrm{~N}_{2}\right) \cdot{ }^{[34]}$

None of these reference data are suitable for direct comparison with our results presented in Table 1; however, a straightforward calculation using the above-mentioned reported uptakes yields an expected value for $\Delta m$ of $\approx 0.017 \mu \mathrm{g} \mathrm{cm}^{-2}$ for the 10x-ZIF-8/PABA substrate. The somehow surprisingly high $1.10 \mu \mathrm{g} \mathrm{cm}^{-2}$ value obtained can be understood in terms of the complex interplay between $\mathrm{N}_{2} / \mathrm{O}_{2}$ uptake at the solid-liquid interface, the intrinsically hydrophobic ZIF-8 microporosity, the mesoporosity stemming from intergrain spaces, and the hydrophilic sites present on the interwoven amino-substituted polymer support.

In summary, we have presented a straightforward and inexpensive strategy to synthesize composite electrodes suitable for enhancing ORR in neutral solutions, with this setting being highly relevant in biofuel cell technologies. Our approach is novel in the sense that ZIF-8 microporous film grown over a CP substrate acts as gas reservoir and, via a preconcentration effect, causes the $\mathrm{O}_{2}$ to be readily available for reduction at the electrode surface. Instead of blocking the surface, the MOF layer covers the CP film providing an efficient pathway for molecular oxygen diffusion (presumably in the hydrophobic intragrain microporosity) as well as the transport of electrolyte ions and solvent (in the intergrain mesoporosity) necessary for effectively achieving the electrocatalytic process. Furthermore, this layer could act as a membrane that protects the active layer toward macromolecular adsorption or contamination that could cause inactivation by fouling in operative conditions and would be of great applicability in compartmentless bioelectrochemical devices as biofuel cells. MOFs and CPs, as a whole, represent a new synergy in materials science. The interplay between the electrocatalytic properties of poly(3-aminobenzylamine-co-aniline) and the oxygen storage capabilities of ZIF-8 at low pressures gave rise to a new interfacial architecture exhibiting a more efficient response toward oxygen reduction as compared to the bare CP. We consider that these results can lead to a new way of looking at CPs as lowcost alternatives for ORR electrocatalysis in mild conditions as well as spark new research directions in the use of MOFs in electrochemical nanoarchitectonics with the attention focused on the rational design of electrocatalytic interfaces with optimized properties.

\section{Supporting Information}

Supporting Information is available from the Wiley Online Library or from the author.

\section{Acknowledgements}

This work was supported by ANPCyT (PICT-2010-2554 and PICT2013-0905), CONICET (PIP 11220130100370CO), UNLP (PPID-X009), Fundación Petruzza, Austrian Institute of Technology GmbH (AITCONICET Partner Lab: "Exploratory Research for Advanced Technologies in Supramolecular Materials Science"- Exp. 4947/11, Res. No. 3911, 28-12-2011) and the Marie Curie International Research Staff Exchange Scheme (IRSES) project HIGRAPHEN (Grant No. 612704). XPS characterization was performed at CIC BiomaGUNE (Spain). Technical support by Danijela Gregurec is greatly acknowledged. M.R., W.A.M., and O.A. are CONICET fellows.

Received: January 20, 2016 Revised: April 18, 2016

Published online: May 20, 2016

[1] Z.-L. Wang, D. Xu, J.-J. Xu, X.-B. Zhang, Chem. Soc. Rev. 2014, 43, 7746.

[2] M. K. Gulbinska (Ed), Lithium-Ion Battery Materials and Engineering, Springer, London 2014.

[3] A. Lavacchi, H. Miller, F. Vizza, Nanotechnology in Electrocatalysis for Energy, Springer, New York 2013.

[4] W. T. Hong, M. Risch, K. A. Stoerzinger, A. Grimaud, J. Suntivich, Y. Shao-Horn, Energy Environ. Sci. 2015, 8, 1404.

[5] M. Shao (Ed), Electrocatalysis in Fuel Cells, Springer, London 2013.

[6] H. Zhong, J. Wang, Y. Y. Zhang, W. Xu, W. Xing, D. Xu, Y. Y. Zhang, X. Zhang, Angew. Chem. Int. Ed. 2014, 53, 14235.

[7] V. G. Khomenko, V. Z. Barsukov, A. S. Katashinskii, Electrochim. Acta 2005, 50, 1675.

[8] C. Q. Cui, J. Y. Lee, J. Electroanal. Chem. 1994, 367, 205.

[9] G. Inzelt, Conducting Polymers: A New Era in Electrochemsitry, Springer-Verlag, Berlin 2012

[10] L. Doubova, G. Mengoli, M. M. Musiani, S. Valcher, Electrochim. Acta 1989, 34, 337.

[11] G. Mengoli, M. M. Musiani, G. Zotti, S. Valcher, J. Electroanal. Chem. Interfacial Electrochem. 1986, 202, 217.

[12] K. Ben Liew, W. R. W. Daud, M. Ghasemi, J. X. Leong, S. Su Lim, M. Ismail, Int. J. Hydrogen Energy 2014, 39, 4870.

[13] Y. Yuan, S. Zhou, L. Zhuang, J. Power Sources 2010, 195, 3490.

[14] H. Furukawa, K. E. Cordova, M. O'Keeffe, O. M. Yaghi, Science 2013 , 341, 1230444.

[15] G. Férey, Chem. Soc. Rev. 2008, 37, 191. 
[16] A. Bétard, R. A. Fischer, Chem. Rev. 2012, 112, 1055.

[17] D. Bradshaw, A. Garai, J. Huo, Chem. Soc. Rev. 2012, 41, 2344.

[18] G. Lu, J. T. Hupp, J. Am. Chem. Soc. 2010, 132, 7832.

[19] K. Díaz, M. López-González, L. F. del Castillo, E. Riande, J. Membr. Sci. 2011, 383, 206

[20] M. Aono, Y. Bando, K. Ariga, Adv. Mater. 2012, 24, 150.

[21] W. A. Marmisollé, D. Gregurec, S. Moya, O. Azzaroni, ChemElectroChem 2015, 2, 2011.

[22] N. Mano, F. Mao, A. Heller, J. Am. Chem. Soc. 2003, 125, 6588.

[23] F. Davis, S. P. J. Higson, Biosens. Bioelectron. 2007, 22, 1224.

[24] H. K. Chaudhari, D. S. Kelkar, J. Appl. Polym. Sci. 1996, 62, 15.

[25] J. Laska, J. Widlarz, Polymer 2005, 46, 1485.

[26] Y. Furukawa, F. Ueda, Y. Hyodo, I. Harada, T. Nakajima, T. Kawagoe, Macromolecules 1988, 21, 1297.
[27] K. S. Park, Z. Ni, A. P. Côté, J. Y. Choi, R. Huang, F. J. Uribe-Romo, H. K. Chae, M. O'Keeffe, O. M. Yaghi, Proc. Natl. Acad. Sci. U S A 2006, 103, 10186.

[28] S. A. Kumar, S.-M. Chen, J. Mol. Catal. A Chem. 2007, 278, 244.

[29] J. J. Low, A. I. Benin, P. Jakubczak, J. F. Abrahamian, S. A. Faheem, R. R. Willis, J. Am. Chem. Soc. 2009, 131, 15834.

[30] K. A. Cychosz, A. J. Matzger, Langmuir 2010, 26, 17198.

[31] X. Liu, Y. Li, Y. Ban, Y. Peng, H. Jin, H. Bux, L. Xu, J. Caro, W. Yang, Chem. Commun. 2013, 49, 9140.

[32] B. Russell, J. Villaroel, K. Sapag, A. D. Migone, J. Phys. Chem. C 2014, 118, 28603.

[33] Z. Lian, L. Huimin, O. Zhaofei, Dalt. Trans. 2014, 43, 6684.

[34] S. Gadipelli, W. Travis, W. Zhou, Z. Guo, Energy Environ. Sci. 2014, 7, 2232. 